\title{
„Haus-CIMT“: Machbar! Aber auch überlegen?
}

Fragestellung: Verbessert eine besondere Form der Constraintinduced Movement Therapy (CIMT), bei der Schlaganfallpatienten ein durch Angehörige supervidiertes Training in der häuslichen Umgebung durchführen, die motorische Funktion?

Hintergrund: CIMT wurde zur Verbesserung motorischer Defizite der oberen Extremität nach einem Schlaganfall entwickelt. Die wesentlichen Elemente sind ein intensiviertes Training für die paretische obere Extremität bei gleichzeitiger Immobilisation des nicht betroffenen Armes. Das initiale Protokoll sah bis zu 60 Therapiestunden bei einer 1:1-Betreuung des Patienten innerhalb von zwölf Tagen vor, spätere Protokolle beinhalteten Modifikationen wie kürzere Therapiezeiten pro Tag, dafür aber einen längeren Gesamtbehandlungszeitraum, oder auch Kleingruppentherapien anstelle von 1:1-Behandlungen. Trotz nachgewiesener Wirksamkeit wird die Methode nur vereinzelt ambulant angeboten. Die aktuelle Studie prüft, ob eine modifizierte CIMT-Version durch niedergelassene Therapeuten

Barzel A, Ketels G, Stark A et al. Home-based constraint-induced movement therapy for patients with upper limb dysfunction after stroke (HOMECIMT): a cluster-randomised, controlled trial. Lancet Neurol 2015; 14: $893-902$ durchgeführt werden kann und wirksam ist.

Patienten und Methodik: In einem randomisierten Design wurden 71 Physiotherapie- und Ergotherapiepraxen ausgewählt, die Schlaganfall-
Patienten (> sechs Monate nach dem Ereignis, leicht bis mäßig betroffen) entweder in Form einer „Haus-CIMT“-Behandlung (37 Praxen, 85 Patienten) oder einer Standardbehandlung (34 Praxen, 71 Patienten) therapieren sollten. Die Therapie erfolgte über vier Wochen und beinhaltete fünf Therapeutenkontakte. In der „Haus-CIMT“-Gruppe wurden Patient und Angehörige instruiert und supervidiert, in der Kontrollgruppe wurde fünfmal behandelt. Das „Haus-CIMT“-Konzept sah täglich ein zweistündiges Training in der häuslichen Umgebung und eine Immobilisierung der nicht betroffenen Hand mit einem Handschuh vor. Motorische Parameter wurden vor und nach den vier Wochen Behandlung sowie sechs Monate später in Form eines Fragebogens (Motor Activity Log, MAL) und eines Funktionstests (Wolf Motor Function Test, WMFT) erhoben.

Ergebnisse: Nach vierwöchigem Training hatten sich beide Gruppen in MAL und WMFT signifikant verbessert. Diese Verbesserung bestand auch noch nach sechs Monaten. Eine Überlegenheit der „Haus-CIMT“-Gruppe ergab sich für den MAL, nicht aber für den WMFT.

Schlussfolgerungen: Patienten der „Haus-CIMT“-Gruppe nahmen subjektiv eine häufigere Nutzung und qualitativ bessere Funktion der betroffenen Hand wahr. Objektive Tests wiesen auf eine vergleichbare Wirksamkeit beider Verfahren hin. Auch chronische Schlaganfallpatienten profitieren von Physio- und Ergotherapie.

\section{- Kommentar von Joachim Liepert, Allensbach}

\section{Ist die Dosis entscheidend?}

In dieser Studie wurde gezeigt, dass ein modifiziertes CIMTKonzept durch niedergelassene Physio- und Ergotherapeuten in Zusammenarbeit mit Angehörigen umgesetzt werden kann. Die investierte Zeit der Therapeuten war vergleichsweise niedrig, sodass das Konzept als nicht personalintensiv angesehen werden kann. Tatsächlich kann man diesen CIMT-Ansatz als einen Paradigmenwechsel betrachten, indem man sich weit von der ursprünglichen therapeutischen 1:1-Betreuung entfernt hat. Patienten der "Haus-CIMT“-Gruppe setzten die betroffene Hand nach eigener Einschätzung zwar häufiger als die Kontrollgruppe ein, bedauerlicherweise führte dieses jedoch nicht zu einer stärkeren Verbesserung motorischer Funktionen. Es stellt sich die Frage, warum ein häufigerer Einsatz der betroffenen Hand nicht auch zu einer stärkeren Funktionsverbesserung führt. Diese Studie kann die Frage nicht beantworten, ein Dosiseffekt ist jedoch die wahrscheinlichste Begründung. Wissenschaftlich herrscht kein Zweifel daran, dass das Ausmaß motorischer Funktionsverbesserungen von Therapieumfang und -intensität abhängt [1]. Auch für die CIMTBehandlung wurde gezeigt, dass ein Training von sechs Stunden pro Tag einem dreistündigen überlegen ist [2]. Vermutlich hätte die "Haus-CIMT"-Gruppe ein noch umfangreicheres und gezieltes Training absolvieren müssen, um sich von der Standardbehandlung zu unterscheiden. Nicht vernachlässigen darf man ein anderes Ergebnis dieser Studie: Auch die übliche therapeutische Behandlung führt zu Funktionsverbesserungen in der chronischen Phase nach Schlaganfall!
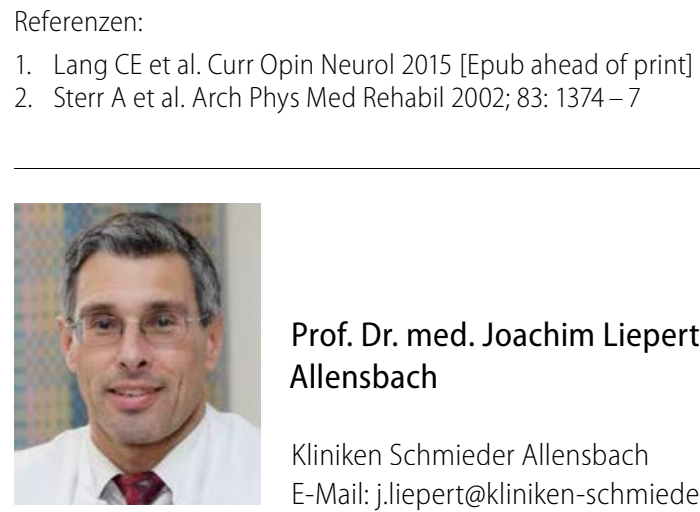

Prof. Dr. med. Joachim Liepert, Allensbach

Kliniken Schmieder Allensbach E-Mail: j.liepert@kliniken-schmieder.de 